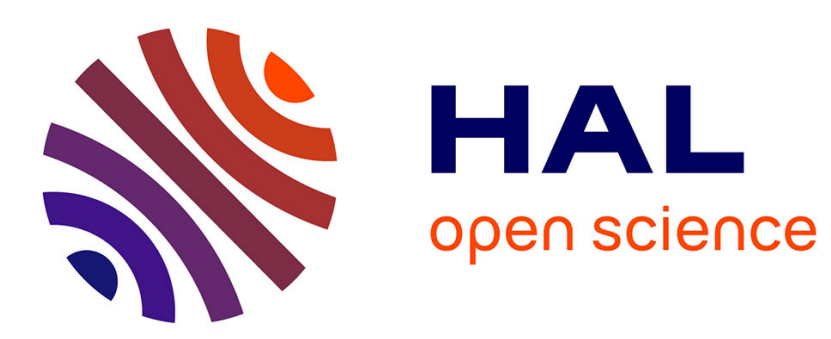

\title{
The evaluation of traditional and early driver learning with simulated accident scenarios
}

Loic Damm, Claudine Nachtergaele, Mohamed Meskali, Catherine Berthelon

\section{To cite this version:}

Loic Damm, Claudine Nachtergaele, Mohamed Meskali, Catherine Berthelon. The evaluation of traditional and early driver learning with simulated accident scenarios. Human Factors, 2011, 53 (4), p323-337. 10.1177/0018720811413765 . hal-00851221

\section{HAL Id: hal-00851221 \\ https://hal.science/hal-00851221}

Submitted on 13 Aug 2013

HAL is a multi-disciplinary open access archive for the deposit and dissemination of scientific research documents, whether they are published or not. The documents may come from teaching and research institutions in France or abroad, or from public or private research centers.
L'archive ouverte pluridisciplinaire HAL, est destinée au dépôt et à la diffusion de documents scientifiques de niveau recherche, publiés ou non, émanant des établissements d'enseignement et de recherche français ou étrangers, des laboratoires publics ou privés. 


\title{
TITLE:
}

\section{THE EVALUATION OF TRADITIONAL AND EARLY DRIVER TRAINING WITH SIMULATED ACCIDENT SCENARIOS.}

\author{
RUNNING HEAD: \\ ACCIDENT SCENARIOS AND DRIVER TRAINING
}

Loïc Damm**, Claudine Nachtergaële*, Mohamed Meskali*, Catherine Berthelon* *IFSTTAR (French Institute of science and Technology for Transport, development and networks), Unité de Recherche Mécanismes d'Accidents, F-13300 Salon de Provence, France

${ }^{* *}$ Current affiliation: Sport and Health Sciences, College of Life and Environmental Sciences, University of Exeter, St Luke's Campus, Heavitree Road, EX1 2LU, Exeter, United Kingdom

Corresponding author: catherine.berthelon@ifsttar.fr

Précis/Short abstract: In France, young people can start driver training from the age of 16, before licensing. Novice, early-trained driver skills were assessed using accident scenarios in a simulator, and compared with those of more experienced and traditionally trained drivers. Pre-license practice could be beneficial when facing challenging situations.

\section{Keywords}

Simulator, driving, training 


\section{Abstract (226 words)}

Objective: We assessed the driving skills of novice, traditionally-trained, novice, earlytrained and experienced drivers to evaluate whether supervised early training could improve young drivers' skills.

Background: The overall representation of young male drivers in car crashes is a recurrent problem in developed countries. In order to prevent this over-representation, France institutes an early driver-training programme from the age of 16 , under the supervision of an adult. However, evidence of the positive effects of this system is still lacking.

Method: Three groups of drivers (12 subjects each) were confronted with five prototypical accident scenarios introduced in a simulated urban circuit. Drivers' response time, speed and vehicle position in the lane were analysed.

Results: No difference was detected across groups regarding obstacle detection, as revealed by the analysis of response times. But in some unexpected scenarios position control by traditionally-trained drivers was more conservative than for more experienced drivers, and early-trained drivers were far more likely to respond with efficient evasive action.

Conclusion: The exposure gained by an early training programme could thus increase the development of visuo-motor coordination and involve better skills in challenging situations.

Application: The supplementary driving experience gained under the supervision of an adult during early training could develop the skills necessary to deal with risky situations. Driving simulators could be used to confront young drivers with a broad ranges of hazardous scenarios not commonly encountered in natural driving. 


\section{Introduction}

Young drivers' crash rates. The high overall representation of young drivers, and particularly male drivers, in car crashes is a well-established fact \{Mayhew et al., 2003, \#87153; Williams and Shabanova, 2003, \#49261; OECD and ECMT, 2006, \#20066; Engströme et al., 2003, \#30026\}. However, the influence of both experience and age, often combined with licensing at a similar age, contribute to confusion in identifying the main factors responsible for this phenomenon \{McCartt et al., 2009, \#83558\}. Exposure must also be considered \{Brown, 1982, \#54976\}. Exposure mainly takes into account the distances driven and the time spent driving, and also includes variables such as the diversity of situations that are encountered. Thus, high exposure during one year of driving is much more beneficial than simply being one year older \{Maycock et al., 1991, \#33942; Waller et al., 2001, \#1601; Mayhew et al., 2003, \#87153\}.

Young drivers versus experienced drivers' skills. It is generally acknowledged that trainees acquire the basics of vehicle handling and traffic rules after 15 hours of lessons \{Hall and West, 1996, \#95935\}. However, the development of cognitive skills is a slower process and includes attentional allocation \{Crundall and Underwood, 1998, \#78917\}, matching between task demands and driving skills \{Brown and Groeger, 1988, \#9631\} and contributes to the driver's potential ability to detect hazards. This crucial ability \{Horswill and McKenna, 2004, \#36171\} improves with experience \{Underwood, 2007, \#48458; Deery, 1999, \#98370\}. Beneficial experience would consist of mapping the relationship between sensorial inputs and hazardous situations. Eye fixation strategies have demonstrated that novice drivers scan a restricted area close to their vehicle 
\{Mourant and Rockwell, \#30687\}, tend to neglect hazardous locations \{Pollatsek et al., 2006, \#14270\} and delay the perception of risky situations \{Finn and Bragg, 1986, \#85366\}. The analysis of drivers' behaviour with different levels of experience in a realistic traffic context appears thus to be relevant to better identify their skill development.

Pre-license practice as a countermeasure. Supervised practice is one of the countermeasures that some countries promote. The graduated driver licensing, which includes a supervised learner's period and an intermediate unsupervised stage in less risky situations was initiated in the USA: it reduces substantially crash rates among 16-year-olds \{Shope et al., 2001, \#80523\}. As attested in Norway, where the minimum age for training is 16, a significant effect of early, accompanied practice is closely linked to the amount of practice $\{$ Sagberg, 2002, \#21361; Sagberg, 2002, \#40814\}. In Sweden, lowering the allowable age for accompanied driving reduced the crash risk by $40 \%$ in the group starting at the age of 16 compared to the group who did not utilize the prolonged training period \{Gregersen et al., 2000, \#73213\}.

In France, before being allowed to take one's driving test, young people have to undergo mandatory training, with twenty hours of lessons with an instructor being the traditional amount. Since 1988, they have been permitted to start early driver training (Apprentissage Anticipé de la Conduite) at the age of 16. The initial apprenticeship is similar (twenty hours of lessons) but the trainee acquires additional experience consisting of driving with an adult for at least 
3,000 $\mathrm{km}{ }^{1}$ before being authorized to take the driving test. Educational meetings are also organized with the driving school. Whatever the type of training, the full driving license cannot be obtained before the age of 18 years.

No systematic approach has been taken to evaluate the impact of this early training on the risk of accidents. A single study, based on questionnaires and interviews, did not observe a clear positive effect PPage et al., 2004, \#24850; Page et al., 2004, \#96871\}. Note that youngsters who engage in early driving training generally have a higher educational background (Chatenet and Leroux, 1999, \#69596 and the sample used by Page et al., consisted mainly of students (78\%) who are traditionally less involved in accidents \{Murray, 1998, \#96054\}, reducing the likelihood that strong effects would be detected. The objective of the present study was to evaluate some of the driving skills of young drivers after early training to emphasize the advantages and/or limitations of this method.

Use of simulator scenarios and driving experience. Simulators today provide a realistic reproduction of driving environments so that the behaviour of drivers immersed in such systems is representative of what can be expected on the road \{Yan et al., 2008, \#84192\}. In this study, simulated accident scenarios i.e. a set of prototypical situations known to generate accidents \{Fleury and Brenac, 2001, \#2064\} were used to test young drivers' skills.

We compared the behaviours of two types of beginners, one group traditionally-trained and the other who underwent early driver training, with

\footnotetext{
${ }^{1}$ Each of them must note his journeys on a dedicated notebook, which could lead to some approximations.
} 
the behaviour of more experienced drivers. We hypothesised that the experience gained during early training could be beneficial when drivers meet a hazardous situation. In particular, the main dynamic parameters of driving, such as the onset of braking before a potential collision and the lateral position of the car on the road, could reveal different types of abilities, and clarify the relation between sensori-motor coordination and experience. A better control of the lateral position of the car was expected among experienced and early trained drivers. On the contrary, the control of braking, when facing a clearly identified obstacle, was not expected to vary across groups.

\section{Methods}

Subjects. Three groups of 12 healthy male volunteers participated in the study. They had normal or corrected-to-normal eyesight. We included only males of relatively similar age to reduce behavioural differences due to age and gender and to make homogenous groups representative of the leading category of drivers involved in car crashes. Drivers were classified as 'experienced' if they had obtained their driving license at least 3 years earlier, had droven a minimum of $50000 \mathrm{kms}$, had their own vehicle and were less than 28 years old (25 \pm 3.1 years). The two other groups were composed of novice drivers who had their driving license for less than one month and did not possess their own car. Twelve traditionally-trained drivers (19.5 \pm 1.3 years $)$ had obtained their license after about twenty hours of lessons with an instructor. Twelve were early-trained drivers (Apprentissage Anticipé de la Conduite in French) (18.5 \pm 0.5 years). The latter followed the same initial apprenticeship of traditionallytrained at a driving school, but they then acquired additional experience 
consisting of driving with an adult for at least $3,000 \mathrm{~km}$. Recruitment of novice drivers was done by the driving school.

The IFSTTAR Internal Ethical Consultative Committee on Biomedical and Behavioural Research approved the research protocol. Participants signed a consent form and an informed commitment form ${ }^{2}$ before the study, and were compensated for their participation (40 euros). They were not susceptible to simulator sickness.

Simulator. The driving study was carried out on the SIM $^{2}$-IFSTTAR fixed-base driving simulator equipped with an ARCHISIM object database \{Espié et al., 2005, \#53364\}. The driving station comprised one quarter of a vehicle (Figure 1). The image projection $(30 \mathrm{~Hz})$ surface filled an angular opening that spans $150^{\circ}$ horizontally and $40^{\circ}$ vertically. The vehicle had an automatic gearbox and was not equipped with rear view mirrors.

Scenarios. The scenario concept used here concerns a group of accidents presenting similarities from the point of view of the chain of events leading to the collision. The sequential analysis method used to group them in the form of scenarios is based on a segmentation of their progression: the situation prior to driving (if information are available); the driving situation (general conditions, driving activity on the route and the section of route leading to the accident site); the accident situation or discontinuity situation, distinguished by an event (e.g.

2 Subjects did not fill out any other questionnaires, but we verified the homogeneity of socio-economical variables. 
arrival to an intersection where another vehicle arrives from the right or the left) or kinematic conditions (e.g. too high speed approaching a difficult bend) indicating a shifting over to a critical situation; the emergency situation, where only extreme manoeuvres could eventually prevent collision; and the collision situation, which includes the impact and its consequences \{Fleury and Brenac, 2001, \#2064; Brenac et al., 2003, \#90808\}. Instead of focusing our study on the measure of a particular ability, we used such scenarios in order to test the influence of experience on the ability to detect the shift over to a critical situation and to perform a coordinated response to avoid an accident.

The scenarios were selected on the basis of their frequency in accident data. They were spatio-temporally implemented using data from the Detailed Accident Study collected by the research unit Mechanisms of Accidents (MA) at IFSTTAR. These highly precise data provided the description of the infrastructure and time positioning of the vehicles and/or pedestrians involved in an accident. Note that only data from the accident situation (discontinuity situation) to the collision situation were reproduced \{Berthelon et al., 2008, \#91644\}.

1. Overtaking scenario: The driver is in the right-hand lane of a straight urban main road with three lanes, two lanes being allocated to his travel direction. A vehicle overtakes him at a speed of $10 \mathrm{~km} \cdot \mathrm{h}^{-1}$ greater than his. When this vehicle is positioned $20 \mathrm{~m}$ in front of the subject, it starts to pull back in ahead of him while slowing down. Three seconds later, it has completely merged into the right-hand lane in front of the subject, and adopts a speed of $30 \mathrm{~km} \cdot \mathrm{h}^{-1}$.

2. Pedestrian scenario: a pedestrian hidden by a bus parked on the righthand side of the carriageway suddenly crosses the carriageway in front of the bus. The pedestrian appears in the driver's field of vision $2.2 \mathrm{~s}$ before the car reaches him.

3. Opposite vehicle crossing scenario: The subject is driving in a straight line on a carriageway with two lanes separated by central marking and carrying two-way traffic. Another vehicle at some distance in front of the driver gets ready to turn left into a petrol station. This vehicle starts to cross the opposite lane when the subject's vehicle is at a distance of $25 \mathrm{~m}$ 
from the potential point of impact. It executes its manoeuvre at low speed, $8 \mathrm{~km} \cdot \mathrm{h}^{-1}$.

4. Left crossroads scenario: the subject's vehicle approaches a crossroads. At $25 \mathrm{~m}$ from the intersection, a vehicle coming from the left starts to slow down for $2 \mathrm{~s}$, adopting a low speed of $10 \mathrm{~km} \cdot \mathrm{h}^{-1}$, and then it decelerates again for another $2 \mathrm{~s}$ to stabilize its speed at $5 \mathrm{~km} \cdot \mathrm{h}^{-1}$ when reaching the crossroads. This vehicle does not have priority and drivers could suppose that it will stop. The vehicle is visible before the subject arrives at the intersection.

5. Parked vehicle scenario: a vehicle parked on the right-hand side pulls out of its parking space as the subject has just turned left at an intersection and is speeding up. This manoeuvre is performed when the subject is around $25 \mathrm{~m}$ from the vehicle and corresponds to a time of $2.5 \mathrm{~s}$ before the collision with an immobile obstacle.

Procedure. Participants were trained to drive the simulator. Then, their task consisted of driving, at $50 \mathrm{~km} \cdot \mathrm{h}^{-1}$ along a realistic (varied infrastructures, signs, traffic lights, buildings...) urban circuit. They drove the circuit twice. The five accident scenarios were introduced in a counterbalanced order in these two trips. Each trip lasted approximately eight minutes. A few independent vehicles with non-conflicting behaviour were also driven along the circuit and no information was given regarding the events to come.

\section{Dependant variables and statistical analysis.}

First, at the starting point for each scenario, named T0, we checked that the speed instructions $\left(50 \mathrm{~km} \cdot \mathrm{h}^{-1}\right)$ were followed (Student's t-test, comparison to a theoretical mean) and the number of collisions was listed for each group of participants. Then, for each scenario, the descriptive parameters of overall driver behaviour were classified as obstacle avoidance (by changing lateral position and/or braking) or collisions. The response time (RT) corresponded to the time difference between the initiation of a scenario (T0) and the first press on the brake pedal. A time breakdown specified key moments for each scenario (see 
figures). At each key moment, the variables analysed were speed and the lateral positions (LP) of the different mobile elements in the scene, LP was calculated in relation to a reference axis (centre of the line).

Statistical tests were then applied to the data: $\chi^{2}$ test for qualitative variables when frequency conditions were combined. ANOVA assessed differences in the mean response time, and mean speed at the initiation of scenarios as a function of the group. ANOVA with repeated measurements (key moments) assessed differences for speed and lateral position as a function of the group. Statistical significance was set at $\mathrm{p} \leq .05$. Bonferroni post-hoc tests were subsequently used for pairwise comparisons.

\section{Results}

Collision rate, response time and respect for instructions. A total of 8 crashes were noted across all scenarios. The traditional group was responsible for half of the collisions whereas the two other groups totalled one quarter of the collisions each. The opposite vehicle crossing scenario caused the most accidents (5), followed by the parked vehicle and hidden pedestrian scenarios ( 2 and 1 collisions, respectively).

Response time did not significantly vary between groups, $F(2,33)=1.21$, $\mathrm{p}=.31$ (traditional $1.36 \pm 0.23 \mathrm{~s}$, early $1.27 \pm 0.22 \mathrm{~s}$, experienced $1.18 \pm 0.2 \mathrm{~s}$ )

The mean speeds measured at the origin of the scenarios (T0), only corresponded to the prescribed speed for the overtaking scenario $(\mathrm{m}=50.13$ $\left.\mathrm{km} \cdot \mathrm{h}^{-1}, \mathrm{t}(35)=0.23, \mathrm{p}<.82\right)$. Speeds were below the instructed value for all other scenarios (hidden pedestrian, $\mathrm{m}=45.9 \mathrm{~km} \cdot \mathrm{h}^{-1}, \mathrm{t}(35)=-4.04, \mathrm{p}<.01$; opposite vehicle crossing, $\mathrm{m}=43.2 \mathrm{~km} \cdot \mathrm{h}^{-1}, \mathrm{t}(35)=-6.71, \mathrm{p}<.01$; left crossroad, $\mathrm{m}=41.3$ 
$\mathrm{km} \cdot \mathrm{h}^{-1}, \mathrm{t}(35)=-7.59, \mathrm{p}<.01$; parked vehicle, $\mathrm{m}=37.4 \mathrm{~km} \cdot \mathrm{h}^{-1}, \mathrm{t}(35)=-9.59, \mathrm{p}<$ $.01)$.

There was no effect of the group on the initial speed $(F(2,33)=0.625, p<$ .54): consequently each group had the same initial clues to build anticipatory behaviour (early $\mathrm{m}=42.7 \pm 7.8 \mathrm{~km} \cdot \mathrm{h}^{-1}$, traditional $\mathrm{m}=43.8 \pm 7.3 \mathrm{~km} \cdot \mathrm{h}^{-1}$, experienced $\mathrm{m}=44.3 \pm 7.4 \mathrm{~km} \cdot \mathrm{h}^{-1}$ ).

\section{Overtaking scenario.}

The start of this scenario (T0, Figure 2) was defined when the obstacle vehicle, in an overtaking manoeuvre in the left-hand lane, was located $20 \mathrm{~m}$ ahead of the driver. This scenario was one of the most time demanding: the Time-To-Collision was $-1.5 \mathrm{~s}$ at T0 (TTC corresponds to the time remaining until the distance between observer and obstacle would become zero, if the current speed of approach is maintained, \{Barbet et al., 2006, \#22002\}).

Every driver, except one from the traditional group, braked after T0. Two main strategies can be observed when the vehicle merged back into the lane of the participant: remaining behind it or changing lane toward the left (table 1). Two cells had expected count less than 5 , so an exact significance test was selected for Pearson's chi-square. Experienced and early groups reacted similarly, adopting a lane changing strategy, $\chi^{2}(1, N=24)=.889, p=.32$. Conversely traditional significantly differed from experienced and often chose to maintain a straight trajectory, $\chi^{2}(1, \mathrm{~N}=24)=4.44, \mathrm{p}=.045$. 


\begin{tabular}{c|ccc}
\hline & Early & Traditional & Experienced \\
\hline Maintain of & & & \\
trajectory & 8 & 10 & 5 \\
Changing lane & & & \\
toward the left & 4 & 2 & 7 \\
\hline
\end{tabular}

Table 1 : Overtaking scenario. Strategies adopted as a function of the groups.

The analysis of speed profiles (Figure 2) did not reveal any difference between groups $F(2,33)=2.55, p=.10$, but showed an effect of time, $F(4,132)=107.08, p$ $<.001$, and a combined effect of group and time, $F(8,132)=3.31, p<.01$. Posthoc, pairwise comparisons with Bonferroni correction showed that speed decreased between T1.1 and T3, $\mathrm{p}<.001$, reached a minimum at T5.3, $\mathrm{p}<.001$, before increasing at the end of the scenario, T10.1, $\mathrm{p}<.001$. Post-hoc tests showed that at T10.1 the experienced group reaccelerated more than traditional : they seem to have considered the scenario to be over earlier and reintegrated themselves into the natural traffic flow.

A group effect, $F(2,33)=6.06, p<0.01$, and a key moment effect, $F(4,132)=$ 18.67, $\mathrm{p}<.001$, were noted on lateral position (Figure 2). Bonferroni post hoc test showed LP globally deviated to the left from T5.3, p $<.001$; LP of experienced was significantly more to the left of the lane $(\mathrm{m}=103 \mathrm{~cm})$ than LP of the traditional group $(\mathrm{m}=41 \mathrm{~cm} ; \mathrm{p}<.004)$, but LP of early $(\mathrm{m}=75 \mathrm{~cm})$ did not significantly differ from LP of the other groups. A cross-effect of group and key moments, $\mathrm{F}(8,132)=5.01, \mathrm{p}<.01$, and associated post hoc tests indicated a significant deviation of experienced drivers' trajectory from T3. At T5.3, i.e. $1.4 \mathrm{~s}$ 
after the obstructing vehicle finished merging, drivers in the experienced group were completely in the left lane of the road $(p<.01)$. This position did not differ from the position of drivers in the early group but was significantly more to the left than the position of traditional drivers (at T5, $\mathrm{p}<.056$; at T10.1, $\mathrm{p}<.001$ ). Thus the experienced group often changed lanes and tended to adjust their strategy more according to the obstacle that interfered with their progression. Even if there was no clear difference between the early and traditional groups, the strategy of the former appeared to be more closely related to the strategy of experienced drivers.

Therefore, the way drivers managed the risky situation differed across groups, as revealed by lateral position discrepancies, but the latency detection of the obstacle and the deceleration that followed it were quite similar.

\section{Pedestrian scenario.}

The pedestrian could be seen $2.2 \mathrm{~s}$ before the participant arrived level with him or her (T0, Figure 3). Here, 56\% of drivers (8 experienced, 6 early, 6 traditional) braked. Most of them (27) kept a quasi-rectilinear trajectory, 5 drivers clearly reoriented their vehicle toward the right (1 traditional, 2 early and 2 experienced) and 4 drivers ( 2 traditional, 1 early and 1 experienced) inappropriately deflected their trajectory to the left, ending in a collision for one driver in the traditional group.

Speed profiles did not exhibit any differences between groups, $F(2,33)=$ $0.04, \mathrm{p}=.96$, but a time effect was noted, $\mathrm{F}(2,66)=55.9 \mathrm{p}<.001$. Post-hoc Bonferroni comparisons showed that speed decreased from T0 (45.5 km.h-1) to 
T2.5 (25.4 km.h-1, p $<.001)$, then regained a value at T5.1 that was not statistically different from the initial one (41.9 km.h-1, p = .15) (Figure 3).

Analysis of LP was restricted to drivers whose trajectory fitted the constraints of the scenario (straight or rightward trajectories). LP did not show any effect of group, $F(2,29)=.69, p=.51$, but a time effect, $F(2,58)=48.30$, $p<$ .001 , from T2.5 $(-4 \mathrm{~cm})$ to T5.1 $(-41 \mathrm{~cm})$ with mean LP significantly shifted to the right of the lane, $\mathrm{p}<.001$ (Figure 3). The interaction time and group, $F(4,58)$ $=3.2, \mathrm{p}<.02$, shows that only experienced and early drivers significantly modified their trajectory to the right $(p<.001)$. Conversely, drivers in the traditional group kept a rectilinear trajectory. Experienced and early groups therefore seemed more able to adequately anticipate the movement of the pedestrian, modifying their vehicle's position accordingly. Among drivers in the traditional group, with the exception of those who deviated massively toward the pedestrian, there was no significant adjustment of their position.

\section{Opposite vehicle crossing scenario.}

This scenario (Figure 4) started when the obstacle vehicle began to accelerate to cross the participant's trajectory (at 25 meters from the participant, mean TTC was $-2.5 \mathrm{~s}$ ). An appropriate deceleration was sufficient to escape collision. Confronted with this hazard, most of the subjects (30) maintained a straight trajectory and braked (except for one in each group). The combination of a steady trajectory and of insufficient braking led to an accident for 1 experienced, 2 traditional and 2 early drivers. Six subjects chose an appropriate leftward action: 3 experienced, 2 traditional and 1 early. 
No differences between expert and novice drivers emerged from the analysis of this scenario. Speed and LP did not vary as a function of group (respectively: $\mathrm{F}(2,33)=1.20, \mathrm{p}=.31$ and $\mathrm{F}(2,33)=2.08, \mathrm{p}=1.14)$, only in function of time (respectively: $F(3,99)=113.29, \mathrm{p}<.001$ and $\mathrm{F}(3,99)=8.06, \mathrm{p}<$ .001). Bonferroni post-hoc tests applied to speed showed a significant decrease from T1 (43.2 km.h-1) to T3 (16.22 km.h-1), then an increase (T5, $30.56 \mathrm{~km} \cdot \mathrm{h}^{-1}$; T10, $44.24 \mathrm{~km} \cdot \mathrm{h}^{-1}$; all $\mathrm{p}<.001$ ). Mean LP globally shifted to the left from T0 to T3 (difference of $35 \mathrm{~cm}, \mathrm{p}<.002$ ) and then stabilized.

\section{Left crossroads scenario.}

The obstacle vehicle, visible early on the left-hand side of the crossroads (Figure 5), was an evident clue to predict the upcoming difficulty. At T0, 28 drivers had identified the potential danger and put their foot on the brake. Six other drivers started to brake only when the obstacle vehicle turned out to be a real danger, and 2 did not brake but took their foot off the accelerator pedal.

Speed and LP only varied as a function of time (respectively $F(3,99)=25$, $\mathrm{p}>.001$ and $\mathrm{F}(3,99)=39.71, \mathrm{p}<.01)$ and were analysed with post-hoc tests. Drivers decelerated from T0 (41.26 km.h-1) to T2.7 (24.91 km.h-1; p $<.001)$, and after a stabilization of their speed, accelerated from T4.2 (26.63 $\left.\mathrm{km} . \mathrm{h}^{-1}\right)$ to T8 (43.38 km.h $\left.{ }^{-1} ; \mathrm{p}<.001\right)$. The LP profile shifted significantly to the right from T0 to T8 (differences had a minimal significance of .04) to reach a total difference of $175 \mathrm{~cm}$ to the right.

Parked vehicle scenario. 
The TTC at T0 was $-2.5 \mathrm{~s}$. Note that for a driver staying in his lane, no evasive action was necessary to avoid collision when braking was forceful enough. Most of the drivers braked (except one in each group) and kept a rectilinear trajectory. Only 4 drivers tried an evasive action to the left: 2 of them ( 1 experienced and 1 early) successfully avoided the obstacle whereas the other 2 ( 1 experienced and 1 traditional) crashed.

Speed only varied as a function of time, $F(4,132)=91.65, p<.001$. From T0 (37.45 km.h-1) to T2.4 (21.93 km.h-1), T3.3 (16.14 km.h-1) and T5 (27.35 km.h1) speed regularly decreased, all $\mathrm{p}<.001$ with post-hoc tests; from T5 to T7 speed increased (35.81 km.h-1, p<.001). Measures on LP revealed some differences across groups, $\mathrm{F}(2,33)=4.96, \mathrm{p}<.01$, and the effect of time, $\mathrm{F}(4,132)$ $=8.06, \mathrm{p}<.001$. Bonferroni post-hoc tests indicated that drivers in the traditional group consistently positioned their vehicle more toward the right (17 $\mathrm{cm})$ than drivers in the experienced $(11 \mathrm{~cm})$ or early $(10 \mathrm{~cm} ; \mathrm{p}<.02)$ groups. Globally, trajectories shifted to the left from T0 $(12 \mathrm{~cm})$ to T2.4 $(10 \mathrm{~cm}, \mathrm{p}<.001)$ before shifting back to the initial position at T7 $(12 \mathrm{~cm})$.

\section{Discussion and conclusions}

Young drivers' skills and abilities. The traditionally-trained group was responsible for half of the collisions, and although this result is not statistically significant due to the size of the sample, it could reflect the effect of a lack of experience.

Our scenarios did not reveal any differences across groups regarding reaction times in contrast to what has been observed by other researchers S Sagberg and Björnskau, 2006, \#97973\}, but are in line with the results of Barbet et al. \{Barbet et al., 2006, \#22002\}, who evaluated the ability of young and experienced drivers 
to visually predict an upcoming collision. Conversely, other experiments have shown that the eye fixation patterns of novice drivers makes them less likely to detect potential hazards, e.g. at intersections, and in typical situations such as parked vehicles $\{$ Borowsky et al., 2009\#56830\}, or when the environment is visually complex $\{$ Underwood, $2007, \# 48458\}$. The differences between novice and experienced drivers appear to vary according to the predictability of the upcoming event, demonstrating the benefits of experience on the ability to classify hazards Borowsky et al., 2009, \#56830; Quimby and Watts, 1981, \#30566; Sexton, 2000, \#48590; Wallis and Horswill, 2007, \#47441\}. These apparent discrepancies between the above mentioned results and ours can be easily reconciled if the complexity of the environment and ocular movements are taken into account, indicating that experienced drivers are more effective in the extraction of useful visual information \{Chapman and Underwood, 1998, \#87000\} \{Crundall et al., 1999, \#24433\}. Thus, even if our results do not show clear differences in response times, the small samples of situations used, the absence of distracting events and the low level of traffic could have masked effects that emerge when conditions become more stringent and require a more flexible strategy \{Crundall and Underwood, 1998, \#78917\}. Moreover, our experienced drivers were less than 28 years old with a minimum of only 3 years of practice whereas RT differences were found with experienced drivers who have been driving for more than 10 years BBorowsky et al., 2009, \#56830\} \{Horswill et al., 2008, \#56553\} \{Sexton, 2000, \#48590\} \{Smith et al., 2009, \#41797\} \{Wallis and Horswill, 2007, \#47441\} or more than 20 years \{Quimby and Watts, 1981, \#30566\}. 
Otherwise, avoidance strategies and trajectories (LP) of novice and experienced drivers revealed some differences in motor abilities. Experienced drivers exhibited an active control of their lateral position during the overtaking and pedestrian scenarios. Early-trained drivers' lateral positions were close to those of experienced drivers, as opposed to the straight trajectory of traditionally-trained drivers. This difference was particularly visible when the time constraint of the scenario was high and when the future critical event was unpredictable, as in both mentioned scenarios. This apparently better skill of the early trained could be linked their supplementary experience (additional mileage under the supervision of an adult). Traditionally-trained drivers also differentiated themselves from other groups by clearly positioning themselves more toward the right of the lane, notably in 2 scenarios (parked vehicle and opposite vehicle crossing). This could be explained by the fact that novice drivers tend to monitor both the foreground and the right of the vehicle for control of their trajectory $\{$ Smiley et al., 1980, \#54428\}. Moreover, successful completion of the scenarios supposed precise visuo-motor coordination and the development of this ability is poorly documented in the driving context. The interaction between visual strategies and visuo-motor coordination calls for further research, and acute time-demanding actions seem relevant to addressing this question.

Methodological considerations. Using a driving simulator is the only safe way of exposing drivers to dangerous situations. Validation of the simulator's characteristics, however, is vital before interpretation. The first level of simulator validity relies on the correspondence of the simulator's layout and dynamics with those of real vehicles and the environment, referred to as 
"physical validity" \{Blaauw, 1982, \#39952\} which supposes that the simulator's dynamics accurately model those of a car \{Triggs, 1986, \#53430\}. Presently the acceleration, braking and steering values of our simulator were those of an average vehicle. Nevertheless, considering novice drivers' low level of driving task automation $\{$ Patten et al., 2006, \#95287\}, the differences between groups observed here (or lack thereof) might have been more pronounced if the simulator had been equipped with a gear lever and rear view mirrors. The limited number of independent vehicles, apart from those directly involved in the scenarios, might also have limited any differences between groups.

Another level of simulator reliability is "behavioural validity" \{Blaauw, 1982, \#39952\}, e.i the simulator's capacity to elicit the same driver response as a real traffic environment \{Rolfe et al., 1970, \#85368\}. Drivers exhibit more conservative driving in a simulator than on the road $\{$ Klee et al., 1999, \#15421\}. Present results also showed a systematically lower driving speed than the one prescribed despite the wide field of view of the simulator $\left(150^{\circ}\right)$ \{Jamson, 2000, \#16864\}. Relative validity is, however, most of the time sufficient: when approaching a curve, drivers' speed profiles, but not values, are similar on a simulator and on the road $\{$ Godley et al., 2002, \#83806\}. Also, the classification of intersection dangerousness obtained experimentally was congruent with the crash information for their field counterparts \{Yan et al., 2008, \#84192\}. Here, we assumed that absolute validity was not essential since comparisons between groups were made with identical scenarios.

Future directions. Simulators, considered as a teaching method, can be used to increase young drivers' exposure to risky situations. The simulated accident 
scenarios used here, directly derived from field studies, could ideally complement hazard detection training. Such use could help novices to build their perceptual and cognitive experience of the driving situations and to acquire the visuo-motor coordination specific to react. Thus, a training CD-ROM accelerated the development of perceptual and cognitive driving skills \{Triggs and Regan, 1998, \#91170\}, the generalisation of the instructional activities to risky traffic situations in a simulator persisted in real traffic $\{$ Regan et al., 2000,\#72166\} and a video-based road commentary quickly improved hazard perception \{Isler et al., 2009, \#61747\}. Similarly, a simple PC-based training programme can enhance scanning behaviour $\{$ Fisher et al., 2006, \#34884\} and realistic avoidance behaviours can be obtained in a simulator \{Hancock and de Ridder, 2003, \#92404\}. Note that training on a simulator provides feedback on the action, which facilitates the acquisition of expertise \{Duncan et al., 1991, \#403\} and produces positive benefits \{Wang et al., 2010, \#99927\},.

As a conclusion, our challenge with young drivers is to give them as much experience as possible in a limited period of time. By providing them with the opportunity to be confronted with various situations, early training beneficially supplements drivers' exposure. 
Key points

- Prelicense pratice is proposed in France as a countermeasure against the high crash rate of young drivers.

- Driving skills of novice traditionally-trained, novice early-trained and more experienced drivers were assessed in a simulator.

- Subjects were confronted with five prototypical accident scenarios.

- Traditionally-trained drivers' position control was more conservative than experienced, and early trained drivers when facing an obstacle.

- The exposure gained before licensing could contribute to the development of better driving skills necessary to deal with dangerous situations. 


\section{Acknowledgements}

We would like to thank the simulation team at IFSTTAR (LEPSIS: Laboratoire

Exploitation, Perception, Simulateurs et Simulations), notably Isabelle Aillerie and Fabrice Vienne, for designing the displays. 


\section{Authors Biographies}

Loïc Damm is a research fellow at the School of Sports and Health Sciences in Exeter, UK. He obtained his PhD in Neurosciences from the University Pierre and Marie Curie in 2008.

Claudine Nachtergaële is an engineer at the French Institute of science and Technology for Transport, development and networks .

Mohamed Meskali, Phd, French Institute of science and Technology for Transport, development and networks.

Catherine Berthelon is a senior researcher at the French Institute of science and Technology for Transport, development and networks. She obtained her PhD from the University of Provence in 1988. 


\section{Figure legends}

Figure 1. Experimental set-up.

Figure 2. Overtaking scenario.

(a) When the overtaking vehicle is positioned $20 \mathrm{~m}$ in front of the subject, it starts to pull back in while slowing down (T0). At T3, it has completely merged into the right-hand lane and adopts a speed of $30 \mathrm{~km} \cdot \mathrm{h}^{-1}$. The end of the scenario correspond to T10.1.

Key moments (T0, T3 and T10.1) are represented by marks on the curves b and c : T1.1 corresponds to the time when the obstacle vehicle's speed and the participant's speed were identical, T5.3 corresponds to the time when the participant reached a minimal speed.

(b) Average lateral position as a function of time for each group,

(c) Average speed as a function of time for each group.

Figure 3. Pedestrian scenario. (a) The pedestrian shoots out from the right 2.4 seconds before the driver crosses his trajectory (T0). T5 corresponds to the end of the scenario.

Key moments (T0 and T5) are represented by marks on the curves b and c : T2.5 corresponds to the moment when the participant crossed the trajectory of the pedestrian

(b) Average lateral position as a function of time for each group 
(c) average speed as a function of time for each group.

Figure 4. Opposite vehicle crossing scenario. (a) A vehicle turns left into a petrol station and starts to cross the subject's lane at a distance of $25 \mathrm{~m}$ from the potential point of impact (T0). T2.5 corresponds to the moment when the obstacle vehicle had a maximal speed, T5 to the moment when the obstacle vehicle finished crossing the participant's lane, T12 to the end of the scenario.

Key moments (T0, T2.5, T5 and T12) are represented by marks on the curves b and $\mathrm{c}$

(b) Average lateral position as a function of time for each group,

(c) Average speed as a function of time for each group.

Figure 5. Left crossroads scenario. (a) When the subject's vehicle is $25 \mathrm{~m}$ from the intersection, a vehicle coming from the left starts to slow down (T0), From T0 to T2, this vehicle decelerates to reach a speed of $10 \mathrm{~km} . \mathrm{h}^{-1}$. From T2 to T4.2 the vehicle decelerates to stabilize its speed at $5 \mathrm{~km} \cdot \mathrm{h}^{-1}$. T8 is the end of the scenario.

Key moments T0, T2, T4.2 and T8 are represented by marks on the curves b and c, T2.7 corresponds to the moment when the participants reached their minimal speed.

(b) Average lateral position as a function of time for each group

(c) Average speed as a function of time for each group. 
Figure 6. Parked vehicle scenario. (a) The vehicle parked on the righthand side pulls out as the subject is about $25 \mathrm{~m}$ from it (T0). At T5 the vehicle has completely finish his manoever. T7 is the end of the scenario.

Key moments (T0, T5 and T7) are represented by marks on curves, at T2.4 the obstacle and the participant have the same speed. At T3.3 the participant reaches minimal speed. T7 is the end of the scenario.

(b) Average lateral positions as a function of time for each group

(c) Average speed as a function of time for each group. 